\title{
Argumentação e Prova Matemática com Geometria Dinâmica
}

\author{
Márcia Rodrigues Notare - PPGEMat/UFRGS - marcia.notare@ gmail.com \\ Marcus Vinicius de Azevedo Basso - PPGEMat/PPGIE/UFRGS - mbasso@ ufrgs.br
}

Resumo: Neste trabalho se discute a contribuição proporcionada pelo uso do software de geometria dinâmica GeoGebra na solução de problemas de geometria que exigem argumentar e provar em matemática. Denominados de caixas-pretas, os problemas analisados neste estudo se caracterizam pela necessidade de reconstruir configurações geométricas a partir da abstração de propriedades geométricas não visíveis na tela do computador. As argumentações e provas matemáticas elaboradas pelos sujeitos da pesquisa, professores em processo de formação com uso de tecnologias digitais, são analisadas a partir do referencial de Villiers e Hanna sobre argumentação e prova matemática com Geometria Dinâmica e do referencial de Jean Piaget sobre abstração reflexionante. De caráter qualitativo exploratório, os resultados do estudo apontam que a atividade de argumentar e provar matematicamente com geometria dinâmica favorece a compreensão e o estabelecimento de relações entre conceitos de matemática.

Palavras-chave: prova matemática, pensamento lógico-dedutivo, geometria dinâmica

\section{Argumentation and Mathematical Proof with Dynamic Geometry}

\begin{abstract}
This paper discusses the contribution of the GeoGebra dynamic geometry software in solving geometry problems that require argument and proof in mathematics. Named as black boxes, the problems analyzed in this study are characterized by the need to reconstruct geometric configurations from the abstraction of geometric properties not visible on the computer screen. The arguments and mathematical proofs elaborated by the research subjects, teachers in the process of formation using digital technologies, are analyzed from the reference of Villiers and Hanna on argumentation and mathematical proof with Dynamic Geometry and the reference of Jean Piaget on reflective abstraction. With an exploratory qualitative character, the results of the study point out that the activity of arguing and proving mathematically with dynamic geometry favors the understanding and establishment of relations between mathematical concepts.
\end{abstract}

Keywords: mathematical proof, logical-deductive thinking, dynamic geometry

\section{Introdução}

A prova é uma característica essencial da Matemática. Ações como experimentar, formular conjecturas, testá-las e validá-las são processos naturais do fazer matemática. No caso da geometria euclidiana, a argumentação tem papel fundamental para a compreensão e validação de teoremas, que caracterizam a essência axiomáticadedutiva desse campo da Matemática. Assim, pensar em alternativas para trabalhar com prova em sala de aula é uma questão fundamental no âmbito da Educação Matemática. Destacamos que não estamos defendendo o trabalho com provas matemáticas que envolvem excesso de rigor e formalismo, mas o processo de raciocínio lógico-dedutivo como ferramenta essencial para a compreensão da Matemática.

Porém, engajar os estudantes em processo de organização de ideias e encadeamento lógico-dedutivo vem sendo um desafio de décadas nas salas de aula de Matemática. Usualmente, o que se observa é o abandono do trabalho com argumentação 
ou, quando é feito, resume-se à memorização para aprovação nos exames finais (Amado, Sanchez, Pinto, 2015)

Para Hanna e Jahnke (1996), a contribuição potencial da prova na Educação Matemática é a comunicação do entendimento matemático, com o objetivo de encorajar os estudantes a fazer conjecturas, explicar seu raciocínio, validar suas afirmações, além de discutir e questionar seu próprio raciocínio e a argumentação dos demais colegas.

Atualmente, ambientes de geometria dinâmica têm colaborado para o resgate das discussões sobre a importância da prova nas aulas de Matemática. A possibilidade de movimentar figuras construídas com propriedades geométricas que as definem faz realçar regularidades e propriedades importantes no processo de argumentação, que se mantêm estáveis durante o movimento e proporcionam um espaço para a elaboração, teste e validação de conjecturas, etapas importantes do processo dedutivo. Mais do que isso, atividades nesses ambientes provocam nos estudantes a necessidade de provar para comprovar o que está sendo observado e abstraído do processo de experimentação e descoberta.

Nesse artigo, analisamos casos em que são apresentadas soluções propostas por estudantes frente a problemas geométricos que exigem provas matemáticas. As análises contemplam aspectos cognitivos à luz da teoria da abstração reflexionante de Piaget, bem como o papel da geometria dinâmica nesse processo de provar, como propõe Villiers $(1998,2004)$.

$\mathrm{Na}$ sequência do artigo, na primeira parte da seção 2, apresentamos as características de problemas do tipo "caixas-pretas" e abordamos o uso da geometria dinâmica na solução desse tipo de problema. Na segunda parte da seção 2, apresentamos os conceitos de abstração reflexionante e tomada de consciência de Piaget, centrais neste estudo. Na seção 3 apresentamos quatro casos envolvendo propostas de provas geométricas de estudantes. Finalizando o artigo, na seção 4, apresentamos considerações finais, apontando para a perspectiva de construção de critérios que contribuam para a construção de objetos/problemas geométricos que requeiram argumentar e provar em Matemática.

\section{Caixas pretas, Geometria Dinâmica e pensamento dedutivo}

\subsection{Sobre caixas-pretas e Geometria Dinâmica}

Softwares de geometria dinâmica são ambientes que permitem a construção de figuras geométricas a partir de suas propriedades básicas, que quando movimentadas preservam suas características originais, ou seja, são figuras ditas estáveis sob a ação do movimento. O dinamismo e a estabilidade proporcionados pelos ambientes de geometria dinâmica permitem aos estudantes explorar, testar, analisar e conjecturar hipóteses sobre uma dada situação geométrica. A possibilidade de arrastar, característica essencial desses ambientes, torna possível a variação contínua de configurações geométricas, que permite que os estudantes investiguem com certa agilidade a situação geométrica apresentada, criando conjecturas (Villiers, 1998, 2004).

Mas o que torna os ambientes de geometria dinâmica tão mais interessantes que os ambientes de sala de aula clássicos com lápis e papel vai além da manipulação direta das figuras, e sim no fato de que toda figura estável construída é concebida em termos de um sistema lógico da geometria euclidiana (Arzarello et al, 2012).

As figuras SGD [construídas em sistemas de geometria dinâmica] possuem uma lógica intrínseca, como resultado de sua construção, colocando os elementos da figura em uma hierarquia de relações que corresponde ao procedimento de construção de acordo com as ferramentas escolhidas e em uma hierarquia de 
propriedades, e essa hierarquia corresponde a uma relação de condicionalidade lógica. Essa relação é evidenciada no modo "arrastamento", onde o que não pode ser arrastado pela variação dos pontos básicos (elementos) de uma figura construída constitui os resultados da construção. A dinâmica dos dados do SGD preserva sua lógica intrínseca; isto é, a lógica de sua construção. A figura do SGD é o complexo desses elementos, incorporando várias relações que podem ser referidas de forma diferente das definições e teoremas da geometria. (Arzarello et al, p.104, 2012)

Atividades que denominamos de "caixas-pretas" caracterizam-se por apresentar aos estudantes uma situação geométrica, normalmente relacionada a um teorema clássico da geometria euclidiana, na qual os passos de construção e propriedades geométricas utilizadas são omitidos dos estudantes. Estes têm acesso à construção geométrica finalizada e aos pontos livres da construção, que podem ser arrastados livremente para explorar e observar as regularidades da construção, que permanecem estáveis na variedade de configurações que surge pelo movimento contínuo dos pontos. A partir desse processo de exploração, o estudante deve abstrair as propriedades que foram impostas na construção, para construir uma situação geométrica que apresente o mesmo comportamento que a figura originalmente observada e explorada.

Este processo de construção corresponde ao que chamamos de "abrir a caixapreta". Nesse processo de "abrir", o estudante é levado a vivenciar etapas inerentes ao processo de argumentação, como explorar, conjecturar, testar e validar. Finalmente, propõe-se ao estudante que argumente e prove as propriedades geométricas observadas e utilizadas na sua construção, para explicar a si mesmo (e aos demais) o entendimento da situação e compreender o encadeamento lógico-dedutivo da argumentação.

A Figura 1 ilustra uma situação geométrica que pode ser caracterizada como uma caixa-preta, se não revelados os passos de construção. Nessa situação, temos um paralelogramo construído (quadrilátero azul) que possui três pontos livres e um ponto dependente (determinado pela intersecção de retas paralelas) e que, portanto, não tem liberdade de movimento.
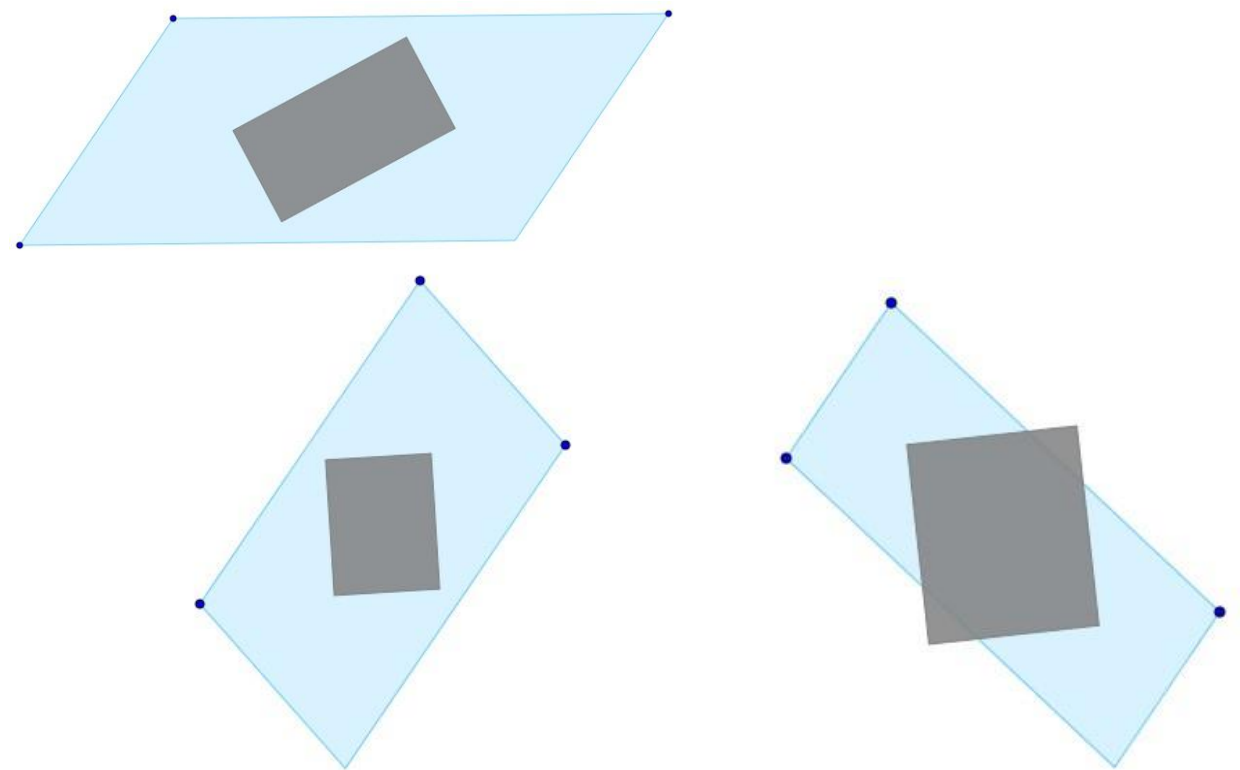

Figura 1: Exemplo de caixa-preta em ambiente de geometria dinâmica. Fonte: Acervo do autor.

A partir do paralelogramo azul, constrói-se o quadrilátero cinza, não revelando as propriedades geométricas impostas nessa construção. O estudante pode arrastar livremente os três pontos livres, em um processo de exploração e observação da 
construção em distintas configurações geométricas (como ilustra a Figura 1), para elaborar conjecturas a respeito do quadrilátero cinza: que tipo de quadrilátero é esse? que regularidades é possível observar como estáveis nas diferentes configurações? que propriedades esse quadrilátero possui? como foi construído? por que possui tais características? como provar que essas características sempre são válidas, independentemente das configurações particulares observadas?

A exploração dinâmica dessa situação geométrica sugere que o quadrilátero cinza é sempre retângulo, permanecendo com essa característica em qualquer configuração do paralelogramo azul, ou seja, é uma regularidade que salta aos olhos quando movimentamos os pontos livres da construção e que, dificilmente seria observada em uma apresentação estática em papel. Temos aqui uma coleção de múltiplos exemplos que realçam essa característica e que fortalecem a possível validade dessa conjectura.

Porém, a identificação de regularidades na construção e elaboração de conjecturas é apenas uma primeira etapa no processo de argumentação e prova e, em especial, no processo de "abrir a caixa-preta". Agora, o desafio é "descobrir" como o quadrilátero cinza foi construído, ou seja, quais propriedades geométricas foram impostas na construção para que o mesmo fosse retângulo.

Além disso, embora seja possível validar de modo geral uma conjectura por meio da observação de regularidades continuamente estáveis em ambiente de geometria dinâmica (por exemplo, a conjectura de que o quadrilátero cinza é sempre retângulo), ainda não há uma explicação satisfatória sobre porque essa conjectura é verdadeira (Villiers, 1998, 2004). O dinamismo do software apenas confirma que a conjectura é válida, mas a argumentação lógica-dedutiva, apoiada em propriedades geométricas e no encadeamento lógico das ideias, é uma etapa importante para o entendimento do porque essa conjectura é válida. O ambiente dinâmico tende a não deixar dúvida de que a propriedade é válida, mas por que?

A Figura 2 ilustra a "caixa-preta" aberta. É possível observar duas retas paralelas (pontilhadas) que determinam lados do paralelogramo. O retângulo é obtido pelos pontos de intersecção das bissetrizes internas do paralelogramo. Desvendar essas propriedades geométricas corresponde a compreender o sistema lógico de construção da figura, o que estabelece uma relação direta entre o mundo das construções da geometria dinâmica e o mundo teórico da geometria euclidiana (Arzarello et al, 2012).

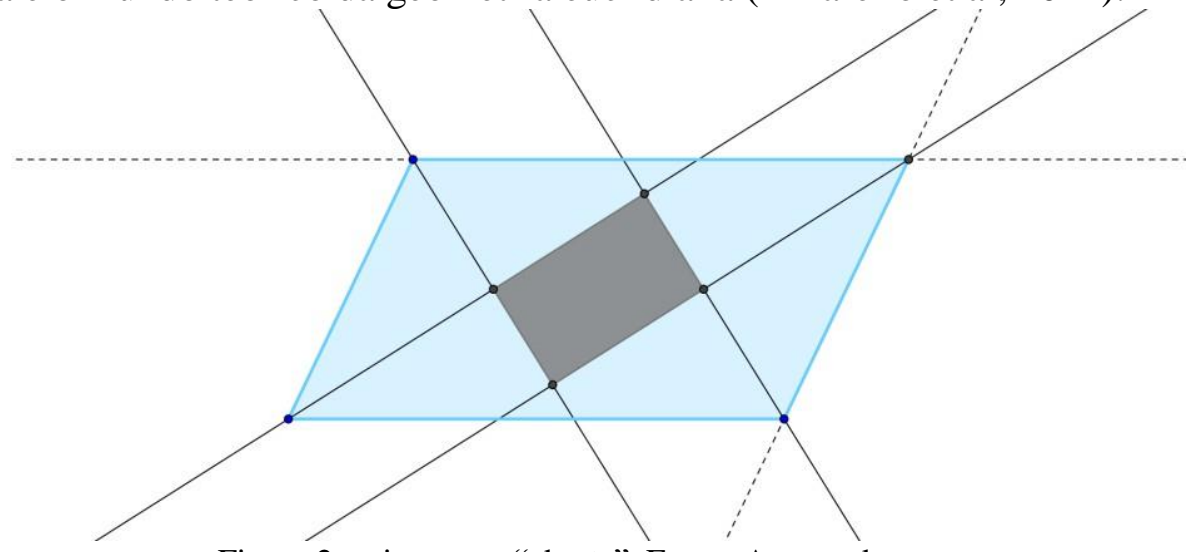

Figura 2: caixa-preta "aberta". Fonte: Acervo do autor.

Nesse processo, encaminha-se à etapa seguinte: por que as bissetrizes de um paralelogramo qualquer determinam um quadrilátero que é sempre retângulo, os seja, possui lados opostos paralelos e congruentes e quatro ângulos congruentes e, portanto, retos? 


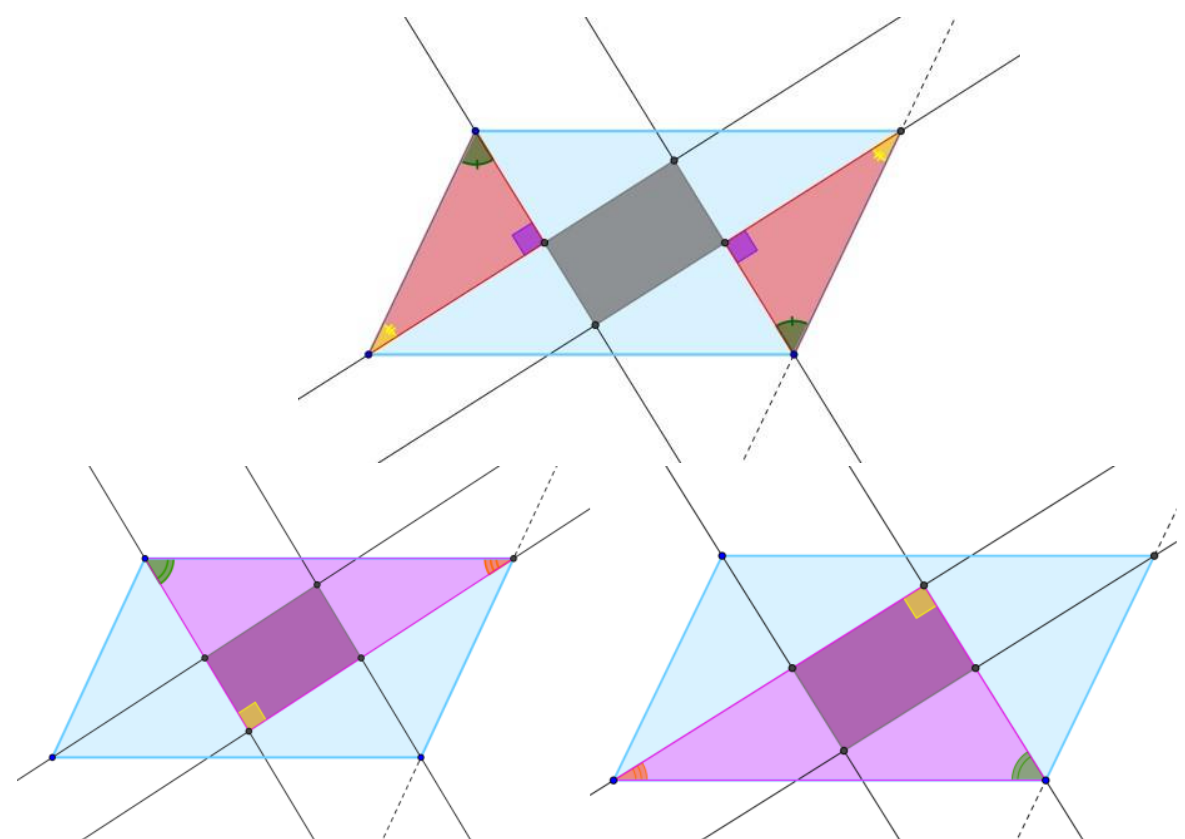

Figura 3: caixa-preta "aberta" com elementos geométricos destacados. Fonte: Acervo do autor.

A Figura 3 destaca elementos geométricos não explícitos na construção, que são cruciais no processo de prova das propriedades observadas, e que permitem o entendimento e a explicação para as conjecturas.

\subsection{Sobre abstração reflexionante e tomada de consciência}

De acordo com a perspectiva teórica piagetiana, o desenvolvimento é um processo que nunca termina (Lourenço, 1994). De forma similar, o processo de construção de conhecimentos de matemática não se esgota na ação sobre objetos, na abstração de propriedades ou no estabelecimento de relações entre conceitos matemáticos. Porém, esses elementos - ações, abstrações e relações entre conceitos constituem bases fundamentais para o desenvolvimento do pensamento dedutivo sendo este, por sua vez, essencial em procedimentos que requeiram demonstrações matemáticas.

Segundo Piaget (1973)

"O papel inicial das ações e experiências lógico-matemáticas, longe de impedir o ulterior desenvolvimento de pensamento dedutivo, constitui, ao contrário, a preparação necessária, e isto por duas razões. A primeira é que as operações mentais ou intelectuais, que participam dos processos de raciocínio dedutivo subsequentes, originam-se das ações. São ações interiorizadas e, uma vez que tenham sido interiorizadas e coordenadas, será o suficiente. Então experiências lógico-matemáticas na forma de ações materiais não serão mais necessárias e a dedução interiorizada será suficiente. A segunda razão é que as coordenações das ações e a experiência lógicomatemática, enquanto se interiorizam, proporcionam a criação de uma variedade particular de abstração que corresponde precisamente à abstração lógica $e$ matemática." (Piaget, 1973. p.2)

As provas em matemática não se originam de abstrações físicas de objetos, as chamadas abstrações empíricas. Tais provas são possíveis a partir de abstrações lógicomatemáticas do tipo abstrações reflexionantes, as quais se caracterizam por,

De um lado, esta abstração 'reflete' (no mesmo sentido que um refletor ou projetor) tudo que estava em um plano inferior (por exemplo, esse plano das ações) e projeta para um plano superior, o do pensamento ou representação mental. Por outro lado, é uma 'abstração reflexionante' no sentido de uma reorganização da atividade mental que reconstrói em um nível superior tudo que foi extraído da coordenação das ações." (Piaget, 1973, p.2) 
Assim, serão as ações e as coordenações dessas ações sobre os problemas geométricos que dão o suporte necessário para as abstrações reflexionantes que podem contribuir para que os estudantes validem suas conjecturas e consigam produzir deduções e provas matematicamente corretas. O uso dos recursos de geometria dinâmica propicia que o estudante construa objetos geométricos, realize movimentos e combinações. Essas construções, movimentos e combinações, constituem as ações que permite aos estudantes testar suas hipóteses a respeito do próprio processo de construção das caixas-pretas.

Porém, no processo de prova em Matemática, também devemos considerar o conceito de abstração refletida, caracterizada como uma abstração reflexionante que se tornou consciente para o estudante. Pelo fato de não termos sempre consciência do raciocínio que utilizamos, a abstração reflexionante não leva necessariamente a uma tomada de consciência. (Montangero e Maurice-Naville, 1998). Em termos de pensamento lógico-matemático, a abstração refletida encontra-se em um patamar superior em relação à abstração reflexionante e esse conceito, por sua vez, coloca em questão o papel da tomada de consciência no processo de provar em Matemática.

Constata-se a partir de casos envolvendo o estudo das caixas-pretas, que, num primeiro momento de exploração dos recursos de geometria dinâmica, predomina o "fazer" sobre os observáveis do objeto, e que tais ações são desenvolvidas no sentido de um "fazer para compreender" (Basso, 2003). Não raro, os conceitos nesse processo de "fazer" são indiferenciados e não suficientemente coordenados entre si. Já a tomada de consciência é o resultado decorrente das explorações sobre os objetos, ou seja, pensar sobre o próprio "fazer" e que pode conduzir a transformação de um "fazer para compreender" em um "fazer e compreender". Esse processo não se esgota necessariamente em uma única etapa do tipo "fazer-compreender". Pode, por outro lado, se estender como na dinâmica "fazer-compreender" e "compreender-re-fazer" representada na Figura 4.

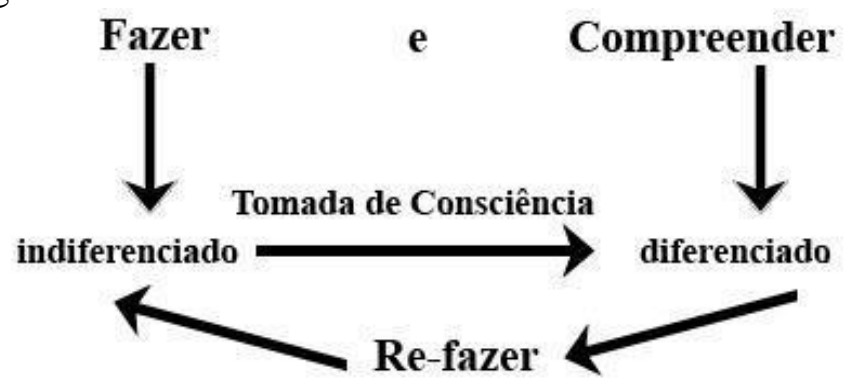

Figura 4: fazer e compreender fazendo. Fonte: Acervo do autor.

No que segue, vamos analisar como professores em processo de formação com uso de tecnologias digitais chegam ao processo de prova em atividade de exploração de caixa-preta.

\section{Procedimentos e análise dos casos}

Nessa seção, analisamos o processo de exploração da caixa-preta discutida na seção 2 por professores em formação para o uso de tecnologias digitais em sala de aula de Matemática. A atividade de "abrir a caixa-preta" é uma das diversas atividades exploradas e discutidas com os professores que cursam o Mestrado em Ensino de Matemática da UFRGS. Foram analisadas as soluções dos 13 professores matriculados. Identificamos quatro categorias de soluções, definidas pelos diferentes patamares de compreensão do processo de prova em geometria e do processo de tomada de consciência da noção de argumentação lógica-dedutiva: 1 . identificação de regularidades e propriedades sem descrição do procedimento; 2. correção na construção 
e descrição do procedimento; 3. correção na construção, estabelecimento de conjecturas pertinentes mas argumentação sustentada exclusivamente na manipulação do software e na observação das regularidades, com base em casos particulares; 4. prova geométrica com estabelecimento de raciocínio lógico-dedutivo, com encadeamento lógico de propriedades e elementos.

A primeira categoria foi caracterizada por sujeitos que se limitaram à identificação de regularidades e propriedades da construção, mas não chegaram ao nível de elaborar conjecturas a respeito do quadrilátero cinza. Os sujeitos dessa categoria foram capazes de realizar a construção geométrica de forma correta, mas não deram continuidade ao processo que poderia conduzir à tomada de consciência e, portanto, de compreensão sobre as construções realizadas. Para eles, a ação de construir foi suficiente como solução do problema, não manifestando necessidade de investigar propriedades para o quadrilátero cinza e, menos ainda, de argumentar sobre a validade dessas propriedades. Entendemos que esses sujeitos não realizaram apenas ações puras, pois foi necessária a abstração de propriedades e regularidades geométricas a partir da manipulação da figura, as quais não estavam explícitas. Ou seja, o processo de abrir a caixa-preta teve sucesso, mas não prosseguiu na direção de uma abstração refletida, base para o pensamento dedutivo.

A segunda categoria avança em relação à primeira pelo fato de, além de realizar a construção corretamente, descrever o procedimento de construção (Quadro 1), em uma hierarquia de relações que corresponde à organização lógica implícita nesse processo em ambiente de geometria dinâmica. Identifica-se um avanço na dinâmica "fazer-compreender" que conduz à tomada de consciência das próprias ações, ou seja, sujeitos dessa categoria precisam reconstituir as ações, que caracteriza um pensar sobre o fazer, mesmo que num patamar inferior ao pensamento dedutivo.

Construção caixa-preta

- criar 3 pontos no plano A, B e C

- traçar os segmentos AB e AC

- criar uma reta paralela ao segmento $\mathrm{AC}$, a partir do ponto $\mathrm{B}$, e uma reta paralela ao segmento $\mathrm{AB}$, partindo do ponto $\mathrm{C}$, retas $\mathrm{c}$ e $\mathrm{d}$.

- Marcar o ponto de intersecção das retas c e d, ponto D.

- Construir o quadrilátero ABDC.

- Traçar as bissetrizes dos ângulos do quadrilátero, retas e, $\mathrm{f}, \mathrm{g}$ e $\mathrm{h}$.

- Marcar os pontos de intersecção entre as bissetrizes, pontos E, F, G e H.

- Construir o quadrilátero EFGH.

- Esconder as retas c, d, e, f, g e h, e os pontos D, E, F, G e H.

Quadro 1: categoria 2 - correção na construção e descrição do procedimento. Fonte: Acervo do autor

A terceira categoria identificada foi caracterizada por sujeitos que realizaram a construção corretamente, estabeleceram conjecturas pertinentes sobre o quadrilátero cinza, mas sustentaram suas argumentações na manipulação do software e na observação das regularidades que se destacam nas múltiplas representações provocadas pelo arrastar dos pontos. Um exemplo desse tipo de solução pode ser verificado no Quadro 2. Pelo extrato da argumentação “...sobrepondo as retas $\boldsymbol{m}$ e $\boldsymbol{j}$ teremos um losango, e como é sabido, as diagonais de qualquer losango são perpendiculares entre si....", percebe-se que o sujeito se apoia em uma configuração geométrica particular para justificar a perpendicularidade dos lados do retângulo. Aqui, já temos indícios de argumentação lógica: há reflexões sobre as ações e abstrações de propriedades não explícitas na construção, além da necessidade de "explicar" o porquê das regularidades continuamente realçadas no ambiente dinâmico, porém ainda apoiada em um caso particular. A explicação apresentada ainda não é satisfatória no entendimento do porque 
o quadrilátero é retângulo, ou seja, não há uma prova lógica-dedutiva para essa conjectura, apoiada em propriedades geométricas e no encadeamento lógico das ideias.

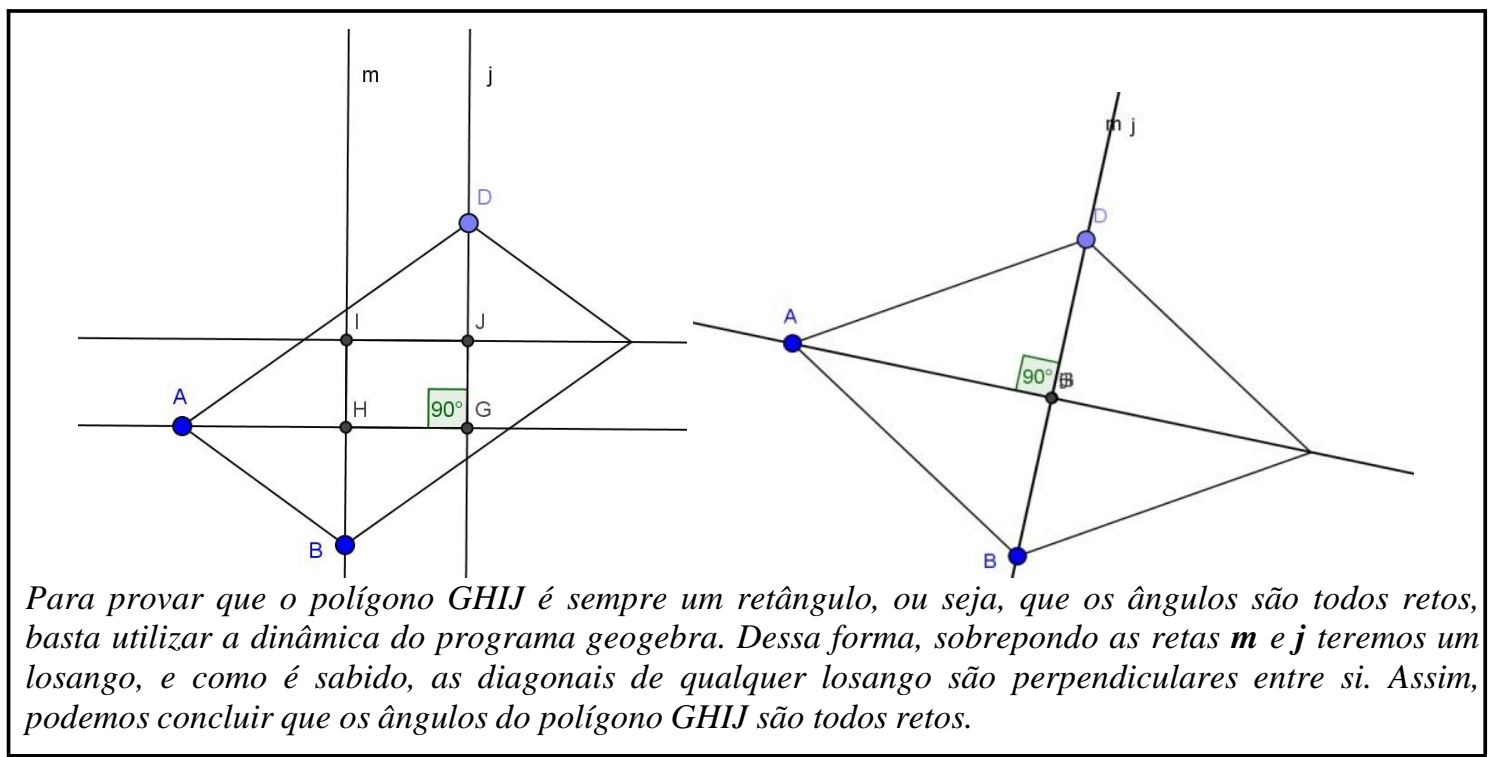

Quadro 2: categoria 3 - conjecturas com argumentação em casos particulares. Fonte: Acervo do autor

A quarta categoria caracteriza-se pelos sujeitos que chegaram ao plano da prova em geometria, estabelecendo raciocínio lógico-dedutivo, com encadeamento lógico de propriedades e elementos que justificam e provam que o quadrilátero cinza é retângulo. Nesses casos, observa-se que os sujeitos são capazes de reconstruir em um nível superior o que foi abstraído da coordenação das ações no ambiente dinâmico do GeoGebra. Há uma reorganização completa da atividade mental, em um patamar que revela a compreensão e controle da situação geométrica. Nesse nível, as ações sobre a figura dinâmica não são mais necessárias, pois já se constituem em ações interiorizadas e coordenadas, que são suficientes para o pensamento dedutivo. No quadro a seguir temos um exemplo de argumentação de sujeito pertencente a essa categoria.

Fatos declarados: $\mathrm{O}$ quadrilátero $\mathrm{ABCD}$ é um paralelogramo. As retas e, f, g, h são bissetrizes dos ângulos $\Varangle \mathrm{A}, \Varangle \mathrm{B}, \measuredangle \mathrm{C}$ e $\measuredangle \mathrm{D}$, respectivamente.

Fato demonstrável: O quadrilátero EFGH é um retângulo.

Demonstração:

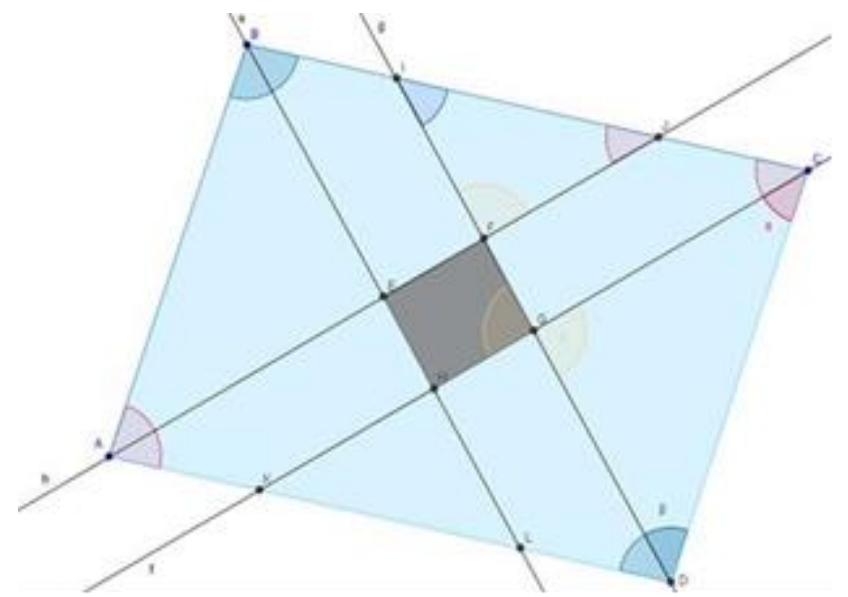

Pelo paralelismo dos segmentos $\mathrm{AD}$ e $\mathrm{BC}$ e a transversalidade das retas $\mathrm{h}$ e $\mathrm{f}$, a primeira passando por A e a segunda passando por B, conclui-se que as retas h e f são paralelas e de forma análoga, as retas e e g são paralelas. Logo EF \| HG e FG || EH, ou seja, o quadrilátero EFGH é um paralelogramo. 
Sejam $\alpha$ o ângulo GCD e $\beta$ o ângulo CDG, pelo paralelogramo $\mathrm{ABCD}$, tem-se que:

$4 \alpha+4 \beta=360^{\circ}$

$\alpha+\beta=90^{\circ}$

$\alpha=90^{\circ}-\beta$

E pelo triângulo GCD, tem-se que $\alpha+\beta+\delta=180^{\circ}$, sendo $\delta$ o ângulo CGD. Então:

$90^{\circ}-\beta+\beta+\delta=180^{\circ}$

$\delta=90^{\circ}$

Assim o ângulo FGH é reto, pois é o.p.v. do ângulo CGD.

De forma análoga para o triângulo $\mathrm{ABE}$ conclui-se que o ângulo $\mathrm{HEF}$ é reto.

Sabendo que e $\|\mathrm{g}, \mathrm{h}\| \mathrm{f}$ e considerando o segmento BC como transversal, pode-se concluir que o triângulo FIJ é semelhante ao triângulo GCD, ou seja, o ângulo EFG é reto. Então, de forma análoga para o triângulo HKL, conclui-se que o ângulo EHG é reto.

Logo, o quadrilátero EFGH é um paralelogramo com os quatro ângulos retos, ou seja, é um retângulo.

Quadro 3: categoria 4 - prova geométrica. Fonte: Acervo do autor

Em todas as categorias, o ambiente de geometria dinâmica constituiu-se em um espaço para ações e coordenações de ações dos sujeitos, dando suporte para as abstrações reflexionantes necessárias para o pensamento dedutivo. Os sujeitos das categorias 1 e 2 encontram-se em nível no qual consideram que a realização da construção correta é suficiente para "provar" as regularidades observadas. No nível em que operam, apoiados em abstrações empíricas e pseudo-empíricas, a partir da ação sobre os observáveis da figura dinâmica, a argumentação lógico-dedutiva não é necessária ou não é alcançada; a validação pela observação das regularidades no ambiente dinâmico, para esses sujeitos, é suficiente. Contudo, reforça-se que essa etapa se constitui base fundamental para o desenvolvimento do pensamento dedutivo e da realização de provas em matemática. Os sujeitos da categoria 3 avançam na atividade de provar suas conjecturas, mas a compreensão do que é uma prova geométrica ainda é incipiente, com argumentações sustentadas por particularidades abstraídas das ações e coordenações das ações sobre a figura dinâmica, e não em propriedades geométricas organizadas com encadeamento lógico. Finalmente, os sujeitos da categoria 4 revelam o nível de pensamento dedutivo, organizando provas consistentes e logicamente organizadas, que não mais dependem de ações e coordenação de ações sobre a figura dinâmica, mesmo que essa etapa tenha sido necessária em algum momento no processo.

\section{Considerações finais}

Finalizando o artigo destacamos que os casos analisados permitem identificarmos elementos relevantes em relação à utilização da geometria dinâmica e, mais especificamente, do GeoGebra, em provas em Matemáticas. As ações proporcionadas pelo ambiente dinâmico, claramente, deram suporte para a identificação de propriedades e regularidades, em todas as categorias observadas, base para o desenvolvimento de provas em geometria. Mesmo os sujeitos que não alcançaram o nível da argumentação lógica, foram capazes de realizar abstrações de propriedades geométricas que não estavam explícitas na construção, revelando o potencial desses ambientes para a dinâmica do fazer e compreender.

Finalmente, destaca-se que este estudo também aponta para a perspectiva de estabelecer-se características para o desenvolvimento de materiais digitais que mobilizem os professores na busca de soluções de problemas geométricos que exijam a argumentação e prova matemática por meio de estratégias que envolvem abstrações reflexionantes e pseudo-empíricas, as quais ampliam as possibilidades de raciocínio lógico-matemático. Além disso, provar em Matemática é fundamental no processo de formação de professores de Matemática e dar atenção a esse aspecto pode contribuir para a atuação docente junto a estudantes do Ensino Básico. 


\section{Referências}

AMADO, N., SANCHEZ, J., PINTO, J. A Utilização do Geogebra na Demonstração Matemática em Sala de Aula: o estudo da reta de Euler, Boletim de Educação Matemática, Rio Claro, v. 29, n. 52, p. 637-657, ago. 2015. Disponível em <http://ref.scielo.org/2z7t4n>. Acesso em 15 maio 2018.

ARZARELlO F., BUSSI M.G.B., LEUNG A.Y.L., MARIOTTI M.A., STEVENSON I. Experimental Approaches to Theoretical Thinking: Artefacts and Proofs. In: Hanna G., de Villiers M. (Eds) Proof and Proving in Mathematics Education. New ICMI Study Series, vol 15. Springer, Dordrecht, 2011. Disponível em <https://link.springer.com/chapter/10.1007/978-94-007-2129-6_5>. Acesso online em 5 maio 2018.

BASSO, M.V.A. Espaços de aprendizagem em rede: novas orientações na formação de professores de matemática. Tese (doutorado) - Universidade Federal do Rio Grande do Sul. Programa de Pós-Graduação em Informática na Educação. Porto Alegre: UFRGS, 2003.

HANNA, G., JAHNKE, N. Proof and Proving. In: BISHOP, A.J. et al. (eds.), International Handbook of Mathematics Education, p. 877 - 908. Dordrecht, Kluwer Academic Publishers, 1996.

LOURENÇO, O.M. Além de Piaget? Sim, mas devagar!... Coimbra: Almedina, 1994. MONTANGERO, J.; MAURICE-NAVILLE, D. Piaget ou a inteligência em evolução. Porto Alegre: Artmed, 1998.

PIAGET, J. Comentários sobre educação matemática. Tradução: Eduardo Britto Velho de Mattos. In: HOWSON, A. G. (Ed.). Developments in mathematical education: proceedings of the 2nd International Congress on Mathematical Education, Exeter, August 29th September 2nd, 1972, Londres: Cambridge University Press, 1973. p. 7987. Disponível em: https://www.ufrgs.br/psicoeduc/piaget/educacao-matematica/> Acesso em 5 maio 2018.

VILLIERS, M. An Alternative Approach to Proof in Dynamic Geometry. In: Lehrer, R. \& Chazan, D. (Eds). Designing Learning Environments for developing Understanding of Geometry and Space. Mahwah, NJ: Lawrence Erlbaum Publishers, pp. 369-393, $1998 . \quad$ Disponível em <https://www.researchgate.net/publication/271728050>. Acesso online em 5 maio 2018 .

VILLIERS, M. Using dynamic geometry to expand mathematics teachers' understanding of proof. International Journal of Mathematical Education in Science and Technology. Taylor \& Francis, 2004, vol. 35, no. 5, 703-724. Disponível em <http://mysite.mweb.co.za/residents/profmd/vanhiele.pdf>. Acesso online em 5 maio 2018. 